\title{
The systemic lupus erythematous in paediatrics: Moroccan experience of a unit of pediatric rheumatology
}

\author{
Kenza Bouayed", K Bouayed, N Echcharaï, N Mikou \\ From 21st European Pediatric Rheumatology (PReS) Congress \\ Belgrade, Serbia. 17-21 September 2014
}

\section{Introduction}

Systemic Lupus Erythematous (SLE) is a chronic autoimmune disease characterized by multi-organ involvement. Its pathogenesis remains controversial. Diagnosis in children is also based on the ARA criteria (American Rheumatism Association).

\section{Objectives}

We propose to report the epidemiological, clinical, immunological, therapeutic and evolutive profile of childhood's SLE through this retrospective study in the Pediatric Rheumatology Service of the Children's Hospital of Casablanca, conducted between December 2001 and January 2014.

\section{Methods}

We report a series of 30 children, including 25 girls, mean age of onset is 12 years, with a range of 7-16 years, the average time from diagnosis is 6 months. The mode of presentation of the disease is classical (general signs, skin and joints involvement) in 56\% of cases, by lupus nephritis in $26.66 \%$ of patients, or a Macrophage Activation Syndrome "MAS" in $13.33 \%$ of cases and lupus nephritis associated with MAS in one patient.

\section{Results}

The clinical picture showed fever in $76.66 \%$ of patients, the frequency of joint locations (90\%), skin (86.66\%), kidney (66.6\%) , hematologic (50\%) lung (36.66\%), gastrointestinal (30\%), neuropsychiatric (26.66\%) and cardiac (23.3\%) involvement.

The hematological involvement was detected in $76.66 \%$ of our patients, an inflammatory syndrome in
83.33\%, immunological disturbances with positive titers of anti- DNAN AC (90\%), ANA (93.6\%), and a reduction of the complement (83.33\%) . False syphilis serology completed by anti $\beta 2$ Glycoprotein 1 antibodies $(26 \%)$ and anti- cardiolopine were positive in respectively 40, 26 and $20 \%$ of cases. Renal involvement is manifested by renal insufficiency in $43 \%$ of cases, a significant proteinuria in $50 \%$ of cases and prevalence of class IV on biopsy. A case of kikushi fujimoto has been reported as 5 hemophagocytic syndromes.

All patients were treated with systemic corticosteroids and hydroxychloroquine. The use of methylprednisolone bolus was indicated in cases of SAM or severe renal impairment associated with cyclophosphamide / MMF and anti-proteinuric medication.

\section{Conclusion}

The prognosis of pediatric SLE remains unpredictable; however it is attached to the renal, neurological and the occurrence of MAS. We mourn two deaths one by neurological attack and other by renal failure.

\section{Disclosure of interest}

None declared.

Published: 17 September 2014

doi:10.1186/1546-0096-12-S1-P328

Cite this article as: Bouayed et al:: The systemic lupus erythematous in paediatrics: Moroccan experience of a unit of pediatric rheumatology. Pediatric Rheumatology 2014 12(Suppl 1):P328. 